\title{
Análise bibliométrica da produção científica brasileira sobre Tecnologia de Construção e Edificações na base de dados Web of Science
}

\author{
Bibliometric analysis of the Brazilian scientific production \\ on Building and Construction Technologies in the Web of \\ Science database
}

\section{Patrícia Bourguignon Soares \\ Teresa Cristina Janes Carneiro \\ João Luiz Calmon \\ Luiz Otávio da Cruz de Oliveira Castro}

\section{Resumo}

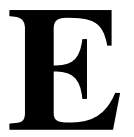

ste estudo trata da análise da produção científica brasileira sobre tecnologias de construção e edificações por meio de indicadores bibliométricos. A área foi escolhida em razão de sua relevância para o desenvolvimento econômico do país. A bibliometria é um método de análise quantitativa para a pesquisa científica. Os dados elaborados por meio dos estudos bibliométricos mensuram a contribuição do conhecimento científico derivado das publicações em determinadas áreas. Os indicadores de produção são úteis para o planejamento e a execução de políticas públicas, e para o conhecimento da comunidade científica sobre o sistema em que está inserida. $\mathrm{O}$ objetivo deste trabalho é estudar a produção científica brasileira na área da construção e tecnologia da construção civil a partir da análise de artigos científicos publicados em periódicos e anais de congressos indexados na base de dados Web of Science (WoS). Os dados foram coletados utilizando como critério de seleção a categoria "Construction \& Building Technology", sendo analisados dados de 910 artigos de autores brasileiros no período de 1982 a 2014. A interpretação dos dados permite confirmar o expressivo crescimento da produção científica brasileira e da grande contribuição da região Sudeste para essa evolução.

Patrícia Bourguignon Soares Universidade Federal do Espírito Vitória - ES - Brasil

Teresa Cristina Janes Carneiro Universidade Federal do Espírito Santo Vitória - ES - Brasil

João Luiz Calmon Universidade Federal do Espírito Vitória - ES - Brasil

Luiz Otávio da Cruz de Oliveira

Castro

Universidade Federal do Espírito Santo

Vitória - ES - Brasil

Recebido em 04/02/15

Aceito em 31/10/15
Palavras-chaves:Tecnologias de construção e edificação. Construção e tecnologia da construção. Bibliometria.Ciência.Tecnologia e inovação.

\section{Abstract \\ This study consists in the analysis of the Brazilian scientific production on Building and Construction Technologies through bibliometric indicators. This field of study was chosen because of its relevance to the country's economic development. Bibliometry is a quantitative analysis method for scientific research. The data elaborated through bibliometric studies measures the contribution of scientific knowledge obtained from publications in specific areas. Production indicators are useful for the planning and execution of public policies and to improve the knowledge of the scientific community about the system that it is a part of. The aim of this study is to assess the Brazilian scientific production in Building and Construction Technology through the analysis of scientific papers published in journals and in proceedings of conferences indexed in the Web of Science (WoS) database. The data were collected using as selection criteria the category "Construction \& Building Technology", and data from 910 articles by Brazilian authors in the period 1982 to 2014 were analysed. The interpretation of the data confirms that there has been a significant growth in Brazilian scientific production and a large contribution of the Southeast region of Brazil to this development.}

Keywords: Construction and building technologies. Construction and construction technology.Bibliometrics.Science. Technology andinnovation. 


\section{Introdução}

Na última década o mundo da pesquisa científica e tecnológica tem passado por transformações profundas, que têm exigido a adoção de novos instrumentos de intervenção e, em decorrência, o tratamento mais criterioso e coordenado da informação. Atualmente, a compreensão e análise dessa nova realidade, de sua dinâmica e complexidade, demanda a produção de indicadores mais robustos, que permitam, de um lado, a apreensão e interpretação de novas formas de produção, difusão e transferência de conhecimentos científicos e, de outro lado, a caracterização de maneira detalhada das capacidades nacionais em C\&T no atual cenário mundial de desenvolvimento científico e tecnológico.

A Ciência e a Tecnologia (C\&T) vêm adquirindo uma enorme importância na sociedade, em parte devido à grande influência que exercem no desenvolvimento econômico, político e cultural dos países. Paralelamente tem surgido a necessidade de avaliar o desempenho da atividade científica e seu impacto na sociedade com o principal objetivo de adequar a alocação de recursos destinados à Pesquisa e Desenvolvimento $(\mathrm{P} \& \mathrm{D})$, considerando um elemento essencial à gestão e ao planejamento científico de qualquer instituição ou país para obter rentabilidade máxima nos investimentos nesse campo (VIOTTI, 2001).

A bibliometria tem sido utilizada como um método de análise quantitativa para pesquisa científica. Os dados estatísticos elaborados por meio dos estudos bibliométricos mensuram a contribuição do conhecimento científico derivado das publicações em determinadas áreas. Esses dados podem ser utilizados na representação das atuais tendências de pesquisa e na identificação de temas para novas pesquisas (SU; LEE, 2010).

Os dados da produção científica, somados à família de indicadores de insumo para a $\mathrm{C} \& \mathrm{~T}$, à cobertura e situação do ensino superior, aos recursos humanos disponíveis em Ciência e Tecnologia, têm contribuído para a análise do desempenho e melhoria da eficiência dos sistemas nacionais da Ciência, Tecnologia e Inovação (FUNDAÇÃO..., 2005). Os dados de produção científica podem ser constituídos com base em uma ampla gama de publicações, como artigos, livros, teses e dissertações. Estima-se que sejam publicados anualmente cerca de 2,5 milhões de artigos em 34 mil periódicos científicos e que esses números não representem a metade do conhecimento científico gerado mundialmente nas universidades e centros de pesquisas (REHEN, 2007).
Segundo a Fundação de Amparo à Pesquisa de São Paulo (Fapesp), os indicadores de produção científica vêm ganhando importância crescente nas últimas décadas como instrumento para análise da atividade científica, em função de sua relação com o desenvolvimento econômico e social. A construção de indicadores quantitativos tem sido incentivada por órgãos internacionais e nacionais de fomento à pesquisa como meio para obter a compreensão mais acurada da orientação e da dinâmica da ciência e como meio de formar e subsidiar o planejamento de políticas científicas, além de avaliar seus resultados (FUNDAÇÃO... 2005).

Analisando-se o setor da construção e tecnologia da construção, a estabilidade econômica e o aumento da competitividade levaram as empresas do setor a pensar na tecnologia como ferramenta de competitividade. A construção e a tecnologia da construção agrega um conjunto de atividades com grande importância para o desenvolvimento econômico e social brasileiro, influindo diretamente na qualidade de vida da população e na infraestrutura econômica do país. Além disso, o setor possui forte relacionamento com outros setores industriais. Ademais, o setor da construção e tecnologia da construção assume importância estratégica como propulsor da atividade econômica, principalmente devido ao volume de recursos que são movimentados à extensa cadeia de fornecedores e à capacidade de ocupação de mão de obra, inclusive com pessoas com baixo grau de escolaridade (SILVA, 2002).

Tendo em vista a escassez de estudos voltados para a análise da produção científica brasileira na área de construção e tecnologia da construção, seria desejável sua intensificação visando contribuir para a elaboração de políticas e estratégias voltadas à superação das carências e limitações do setor, bem como à necessidade de melhor compreender as especificidades de seus processos de desenvolvimento científico, tecnológico e econômico.

Esses motivos, entre outros, ratificam que a área da construção e tecnologia da construção, apesar de sua importância relativa para o setor econômico brasileiro, ainda é caracterizada como um setor tradicional, conservador e tímido quanto à produção científica. Ademais, análise da produção científica e tecnológica sobre de construção e tecnologia da construção é um tema pouco abordado por estudos acadêmicos, não existindo consenso sobre o entendimento desse objeto de estudo, tampouco se configura uma tradição de

176 Soares, P. B.; Carneiro, T. C. J.; Calmon, J. L.; Castro, L. O. da C. de O. 
pesquisa que consolide abordagens e metodologias específicas.

A elaboração de indicadores bibliométricos para o segmento de construção e tecnologia da construção pressupõe uma abordagem multidisciplinar, exigindo um trabalho metodológico minucioso e transparente que permita a produção de um conjunto coerente de indicadores, o que faculta a seus usuários não só uma visão abrangente dessa área como também as limitações inerentes a esses indicadores. Dessa forma, o objetivo do presente trabalho é estudar a produção científica brasileira de construção e tecnologia da construção utilizando dados bibliográficos indexados na base de dados da Web of Science.

\section{Construção civil no Brasil}

A indústria da construção civil é um setor que tem um produto não homogêneo e não seriado, que depende de encomendas, o que implica a produção de um produto único. Uma das características marcantes do setor da construção civil é sua heterogeneidade, seja pela abrangência de atividades, seja pela tipologia das empresas, seja pelas tecnologias e qualificação de pessoal, seja ainda pela dispersão geográfica. É uma indústria atípica com uma dependência recíproca entre o produto e o processo produtivo (CENTRO..., 2009; SOUTO, 2003).

Trata-se de uma indústria composta de uma complexa cadeia produtiva, que abrange setores industriais diversos, tais como mineração, siderurgia do aço, metalurgia do alumínio e do cobre, vidro, cerâmica, madeira, plásticos, equipamentos elétricos e mecânicos, fios e cabos, e diversos prestadores de serviços, como escritórios de projetos arquitetônicos, serviços de engenharia, empreiteiros, entre outros. Abriga desde indústrias de tecnologia de ponta e capital intensivo, como cimento, siderurgia e química, até milhares de microempresas de serviços, a maior parte com baixo conteúdo tecnológico (DEPARTAMENTO..., 2008).

A despeito da heterogeneidade, o setor da construção civil representa grande importância para o desenvolvimento econômico e social do país, destacando-se pela quantidade de atividades que intervêm em seu ciclo de produção, gerando consumo de bens e serviços de outros setores, além de absorver grande parte da mão de obra não especializada (BANCO..., 2010).

Apesar da grande importância da indústria da construção civil para o desenvolvimento econômico e social do país, o setor possui baixo investimento em pesquisa e desenvolvimento
(P\&D), não sendo possível quantificar corretamente esse volume. Possui um histórico de baixa velocidade de difusão de novos recursos tecnológicos, contrapondo-se aos demais setores, nos quais as inovações tecnológicas são rapidamente absorvidas, implantadas e aperfeiçoadas (MARTINS; BARROS, 2003).

De acordo com o Programa Inovação Tecnológica na Construção (PIT) da Câmara Brasileira da Indústria da Construção (CBIC), a Pesquisa e Desenvolvimento (P\&D) na construção civil ocorre com três agentes (PROGRAMA...., 2012):

(a) fabricantes de materiais e sistemas construtivos;

(b) empresas incorporadoras e construtoras; e

(c) universidades e institutos de pesquisa atuantes na área da construção ou em áreas de interface.

Esses agentes são líderes no processo de desenvolvimento e inovação. No entanto, a maioria das inovações tecnológicas nasce no desenvolvimento da indústria, e em alguns desses processos ocorre a participação de pesquisadores associados a universidades e institutos de pesquisas (PROGRAMA..., 2012).

\section{Método da pesquisa}

A bibliometria possibilita a observação do estado da ciência e tecnologia por meio de toda a produção científica registrada em um repositório de dados. É um método que permite situar um país em relação ao mundo, uma instituição em relação a um país, e cientistas individuais em relação às próprias comunidades científicas. Baseia-se na contagem de artigos científicos, patentes e citações. Dependendo da finalidade do estudo bibliométrico, os dados podem ser tanto o texto que compõe a publicação como os elementos presentes em registros sobre publicações extraídos de base de dados bibliográficos, como nome de autores, título, fonte, idioma, palavra-chave, classificação e citações (RAO, 1986; ZHU et al., 1999). A bibliometria pode auxiliar na identificação de tendências de crescimento do conhecimento em determinada disciplina, dispersão e obsolescências de campos científicos, autores e instituições mais produtivos, e periódicos mais utilizados na divulgação de pesquisas em determinada área do conhecimento.

$\mathrm{Na}$ presente pesquisa foram utilizados dados de produção científica brasileira baseada em artigos publicados em periódicos e anais de congressos indexados na base de dados Web of Science da área do conhecimento da construção civil. A Web of Science é uma base multidisciplinar desenvolvida 
pela Thomson Scientific - Institute for Science Information (ISI). Foi escolhida como fonte de dados bibliográficos para avaliar a relação entre autores, instituições, estados, áreas do conhecimento e países dos artigos selecionados. Como critério de seleção dos dados foi utilizado a categoria com dois termos: "Construction \& Building Technology", considerando ser terminologia de busca padrão; e "Brasil".

Foram considerados artigos brasileiros aqueles que pelo menos um autor possua filiação com instituições de ensino ou pesquisa brasileiras. Foram selecionados 910 artigos científicos, sendo $680(74,7 \%)$ artigos publicados em periódicos acadêmicos, $206(22,6 \%)$ publicados em anais de eventos científicos indexados na base de dados, e $24(2,7 \%)$ artigos publicados tanto em periódicos quanto em anais de eventos científicos. Das bases de dados que compõem a WoS, foram utilizadas Science Citation Index - Expanded (SCI); Social Science Citation (SSCI) e Conference Science Citation index (CSCI). A busca foi realizada em janeiro de 2015, abordando o período de 1982 a 2014.

\section{Resultados da pesquisa}

\section{Evolução anual da produção científica brasileira sobre construção e tecnologia da construção}

Na Figura 1 observa-se que o primeiro artigo publicado disponível na base WoS foi de 1982, de autoria de Dao Martinelli et al., da USP de São Carlos. O artigo é denominado "Ferrocement structures by the São Carlos Group (Brazil)"e foipublicado no Journal of Ferrocement -
Bangkok. Entre 1982 e 1993 houve um pequeno número publicações (entre 1 e 7 artigos por ano), porém a partir de 1994 a produção cresceu. A partir de 2007 é possível observar um crescimento significativo em relação aos anos anteriores, ressaltando os picos ocorridos em 2007 (89 artigos) e em 2013 ( 85 artigos). Ressalta-se que em 2014 foram registrados somente 17 artigos nesse estudo em virtude de a pesquisa ter sido efetuada em janeiro de 2015. A amostra pode sofrer alterações devido aos prazos de indexações de periódicos na base de dados da WoS.

A tendência de crescimento expressivo da produção brasileira vem sendo registrada em estudos realizados pela Fundação de Amparo à Pesquisa de São Paulo (Fapesp) em outras áreas do conhecimento, não especificamente da construção e tecnologia da construção. Esse crescimento pode estar associado ao crescimento dos programas e alunos de pós-graduação e à melhoria da qualificação do corpo docente das universidades (FUNDAÇÃO..., 2010).

Dados da Câmara Brasileira da Indústria da Construção (CÂMARA..., 2013) mostram que o setor de construção e tecnologia da construção passou por um período de instabilidade devido à falta de incentivo e baixa disponibilidade de recursos. Somente a partir de 2004 o setor começa a ter um significativo crescimento em função dos aumentos dos investimentos em obras e infraestrutura, através de programas governamentais. Esses fatores podem ter favorecido a busca de estudos sobre construção e tecnologia da construção devido à necessidade de novos desenvolvimentos tecnológicos, o que pode justificar a elevação da produção científica do Brasil, publicada em periódicos e eventos internacionais, ocorrida a partir de 2003.

Figura 1 - Frequência absoluta (n) da evolução da produção científica no Brasil sobre construção e tecnologia da construção no período de 1982 a 2014

Evolucao de Artigos de Autores Brasileiros na Base WoS

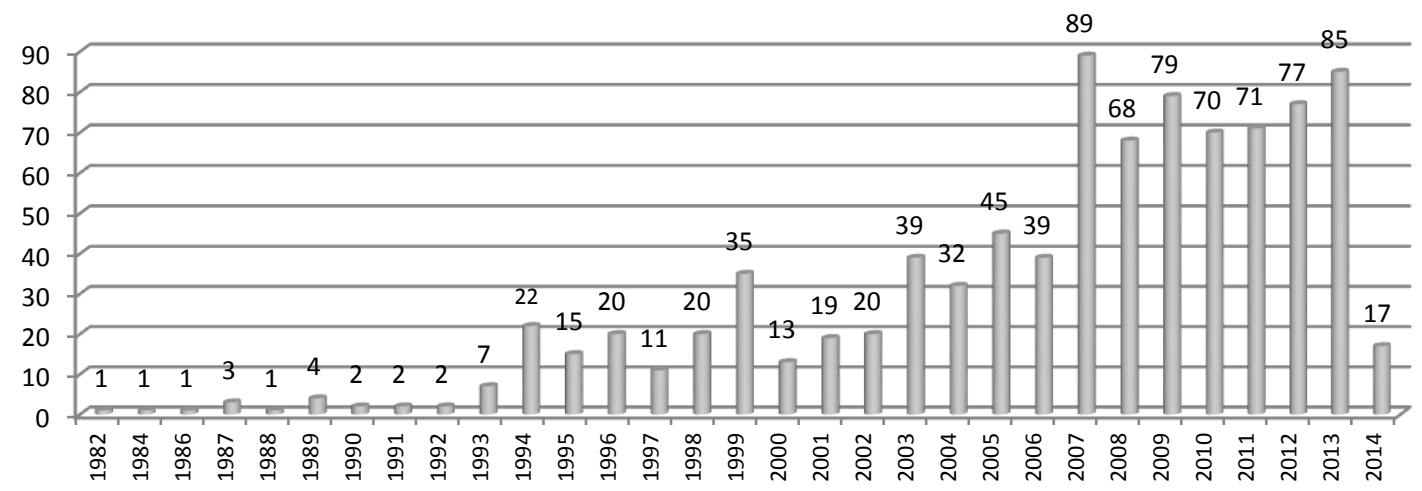

Fonte: Web of Science (2014).

178 Soares, P. B.; Carneiro, T. C. J.; Calmon, J. L.; Castro, L. O. da C. de O. 
Graças a uma política consistentemente favorável para a pós-graduação nos últimos 50 anos, o número de pessoas com diplomas de doutorado no Brasil cresceu de 554 em 1981 para 10.711 em 2008. Além disso, parece haver correlação entre o aumento da produção de literatura científica e o crescente número de pessoas recebendo a titulação de doutorado a cada ano. No entanto, as áreas que apresentam a maior parcela da produção científica brasileira são Medicina clínica, ciências de plantas e animais, ciências agrárias, química e física. As engenharias aparecem em oitavo lugar (THOMSON..., 2013).

A Figura 2 mostra o ranking das instituições brasileiras com maior número de autorias de publicações. Das 23 instituições de ensino superior (IES) com pelo menos 10 autorias na base WoS, $16(70 \%)$ são instituições federais de ensino, quatro (17\%) são universidades estaduais, e três (13\%) são instituições particulares.

A Universidade de São Paulo (USP) ocupa o primeiro lugar, com 214 autorias associadas. Por autoria associada, entende-se um autor de um artigo que informou ser filiado à instituição. Um artigo pode ser contado mais de uma vez, dependendo do número de autores do artigo. Foram identificadas nos 910 artigos selecionados 1.626 autorias, uma média de 1,8 autor por artigo. Os valores que aparecem no Gráfico 2 referem-se à autoria de artigo associada às IES.

Em seguida à USP, aparece a Universidade Federal do Rio de Janeiro (UFRJ), n=112, a Universidade
Federal de Santa Catarina (UFSC), n=101, a Universidade Federal do Rio Grande do Sul (UFRGS), n=65, a Universidade Federal de Minas Gerais (UFMG), n=42, e a Pontifícia Universidade Católica do Rio de Janeiro (PUC-RJ), n=41. A Universidade Federal do Espírito Santo (Ufes) e a Universidade do Vale do Rio Sinos (Unisinos), com 10 autorias cada, encerram o ranking de IES com até 10 autorias.

$\mathrm{Na}$ Tabela 1 é possível verificar que, entre as instituições de ensino superior com até 10 autorias na base WoS na área de construção e tecnologia da construção, nenhuma pertence à região Norte do Brasil. As regiões Sudeste e Sul possuem o maior número de IES (11 e 7 respectivamente) e o maior número de autorias associadas $(561$ e 257 respectivamente). A unidade da federação (UF) com maior número de IES é o Rio de Janeiro, com cinco IES (UFRJ, PUC-RJ, UFF, UERJ e UENF), seguida por São Paulo (USP, Unicamp e UFSCar), Rio Grande do Sul (UFRGS, UFSM e Unisinos) e Paraná (UFPR, PUC-PR e UTFPR), com três IES cada. A UF com maior número de autorias é São Paulo ( $n=275)$, seguida de perto pelo Rio de Janeiro $(n=220)$. Os dois estados da região Sudeste com maior número de autorias são os mais populosos do Brasil e com maior número de edificações. É possível observar que essa tendência ocorre também nas demais regiões: os estados com maior número de autorias associadas são também os mais populosos e os que mais demandam edificações.

Figura 2 - Frequência absoluta de autorias de publicações brasileiras sobre construção e tecnologia da construção associadas às instituições de ensino superior indexadas na base de dados WoS no período de 1982 a 2014

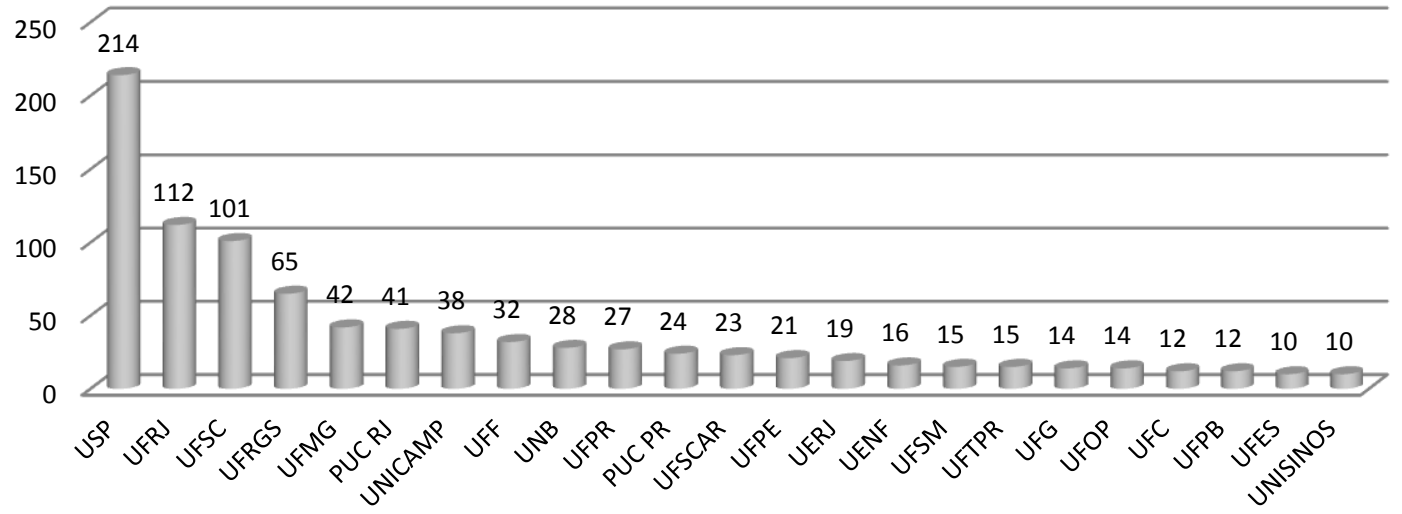

Fonte: Web of Science (2014). 
Tabela 1 - Frequência absoluta de autorias de publicações brasileiras sobre construção e tecnologia da construção associadas às IES por região no período de 1982 a 2014

\begin{tabular}{l|c|c|c|c}
\hline Região & UF & Qt. IES & Qt. Autorias \\
\hline \multirow{4}{*}{ Centro-Oeste } & DF & 1 & 28 \\
\cline { 3 - 5 } & GO & 1 & 14 \\
\cline { 3 - 5 } & Total & $\mathbf{2}$ & $\mathbf{4 2}$ \\
\hline \multirow{4}{*}{ Nordeste } & PE & 1 & 21 \\
\cline { 3 - 5 } & CE & 1 & 12 \\
\cline { 2 - 4 } & PB & 1 & 12 \\
\cline { 2 - 4 } & Total & $\mathbf{3}$ & $\mathbf{4 5}$ \\
\hline \multirow{4}{*}{ Sul } & SC & 1 & 101 \\
\cline { 2 - 4 } & RS & 3 & 90 \\
\cline { 2 - 4 } & PR & 3 & 66 \\
\cline { 2 - 4 } & Total & $\mathbf{7}$ & $\mathbf{2 5 7}$ \\
\hline \multirow{4}{*}{ Sudeste } & SP & 3 & 275 \\
\cline { 2 - 4 } & RJ & 5 & 220 \\
\cline { 2 - 4 } & MG & 2 & 56 \\
\cline { 2 - 4 } & ES & 1 & 10 \\
\cline { 2 - 4 } & Total & $\mathbf{1 1}$ & $\mathbf{5 6 1}$ \\
\hline
\end{tabular}

Fonte: Web of Science (2014).

A região Sudeste possui a maior concentração de autorias de produção científica indexada na WoS na área de construção e tecnologia da construção. A produção científica no Sudeste pode estar associada à grande concentração de instituições de ensino superior, de programas de pós-graduação e programas apoiados por fundações estaduais de ciência e tecnologia, implementados pelas agências de fomentos locais e federais, como o Conselho Nacional de Desenvolvimento Científico (CNPq), a Coordenação de Aperfeiçoamento de Pessoal de Nível Superior (Capes) e a Financiadora de Estudos e Projetos (Finep) (FUNDAÇÃO..., 2010; GREGOLIN et al., 2005). A Figura 3 mostra que entre as agências financiadoras de pesquisas há três fundações estaduais de apoio à ciência e tecnologia da região Sudeste (Fundação de Amparo à Pesquisa do Estado de São Paulo Fapesp, Fundação Carlos Chagas Filho de Amparo à Pesquisa do Estado do Rio de Janeiro - Faperj e Fundação de Amparo à Pesquisa do Estado de Minas Gerais - Fapemig); uma da região Sul (Fundação de Amparo à Pesquisa do Estado do Rio Grande do Sul - Fapergs) e três agências de fomento federais (CNPq, Capes e Financiadora de Estudo e Projetos - Finep), sendo as duas primeiras as maiores financiadoras, com 147 e 110 artigos apoiados respectivamente.

Esses resultados mostram uma provável associação entre financiamento de pesquisa e número de artigos científicos publicados em periódicos e eventos científicos indexados em base de dados internacionais. A participação em eventos no exterior demanda recursos de apoio a viagem e estadia dos pesquisadores, nem sempre possíveis de ser financiados pelas IES a que pertencem e desenvolvem suas pesquisas. A participação em eventos internacionais é uma importante etapa na divulgação de resultados de pesquisa que podem depois ser publicados em periódicos internacionais, de maior visibilidade.

\section{Autores e a produção científica brasileira em construção e tecnologia da construção}

A Tabela 2 mostra o número de publicações científicas por autor. $\mathrm{O}$ pesquisador Romildo Dias Toledo Filho, da COPPE/UFRJ, lidera o ranking com 34 publicações. Seu principal tema de pesquisa é concreto. Em segundo lugar, com 26 publicações, está o Professor Holmer Savastano Junior, da USP, com pesquisas na área de materiais e componentes não convencionais de construção. Em terceiro lugar, com 16 publicações, está o Professor Nathan Mendes, da PUC-PR, que pesquisa sobre energia, calor e umidade em construções, análise de sistemas de climatização e eficiência energética de edificações. Em seguida vem o Professor Sebastião Arthur Lopes de Andrade, da PUC-RJ, que desenvolve pesquisas sobre estruturas. No quinto lugar do ranking, com 10 publicações, encontra-se o Professor Pedro Colmar Gonçalves da Silva Vellasco, da UERJ, que também pesquisa sobre estruturas. Entre os pesquisadores com maior número de publicações internacionais na área, dois são de instituições estaduais de ensino, dois de instituições particulares, e apenas um de instituição federal. Esses resultados mostram que na área de 
construção e tecnologia da construção as pesquisas com parcerias e visibilidade internacionais estão sendo desenvolvidas tanto em instituições públicas como em instituições privadas de ensino.

Sobre as coautorias dos artigos publicados, as principais parcerias são com pesquisadores filiados a instituições de ensino americanas $(n=78,8,6 \%)$ seguida pela Inglaterra e Espanha $(n=39,4,3 \%)$, conforme pode ser observado na Tabela 3 . Lembra-se que um artigo pode ter vários autores e que cada autor conta como uma coautoria. Dos 10 países com maior número de parcerias de pesquisa, seis são países europeus, três americanos, e nenhum é asiático.

Figura 3 - Frequência absoluta de publicações brasileiras sobre construção e tecnologia da construção associadas às maiores agências de financiamento de C\&T indexadas na base de dados WoS

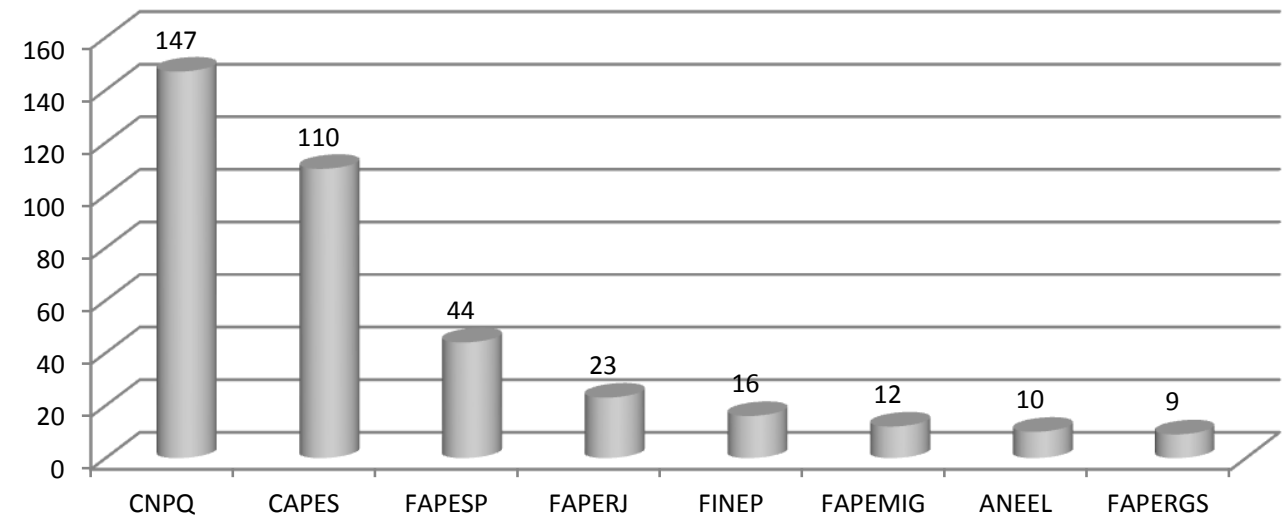

Fonte: Web of Science (2014).

Tabela 2 - Frequência absoluta do número de produção científica brasileira sobre construção e tecnologia da construção por autor com publicações indexadas na base de dados da WoS no período de 1982 a 2014

\begin{tabular}{l|c|c|c}
\hline \multicolumn{1}{c|}{ Autor } & IES & Artigos & $\mathbf{\%}$ \\
\hline Romildo Dias Toledo Filho & UFRJ & 34 & 3,7 \\
Holmer Savastano Junior & USP & 26 & 3,0 \\
Nathan Mendes & PUC-PR & 16 & 1,8 \\
Sebastião Arthur Lopes de Andrade & PUC-RJ & 14 & 1,5 \\
Pedro Colmar Gonçalves da Silva Vellasco & UERJ & 11 & 1,2 \\
\hline
\end{tabular}

Fonte: Web of Science (2014).

Tabela 3 - Frequência do número de coautorias da produção científica brasileira sobre construção e tecnologia da construção por país de origem do coautor no período de 1982 a 2014

Fonte: Web of Science (2014).

\begin{tabular}{l|c|c}
\hline \multicolumn{1}{c|}{ Países } & $\begin{array}{c}\text { Autorias de } \\
\text { Artigos }\end{array}$ & $\begin{array}{c}\text { \% do Total de } \\
\text { Artigos }\end{array}$ \\
\hline EUA & 78 & 8,6 \\
Inglaterra & 39 & 4,3 \\
Espanha & 39 & 4,3 \\
Portugal & 36 & 4,0 \\
França & 27 & 3,0 \\
Alemanha & 12 & 1,3 \\
Austrália & 11 & 1,2 \\
Holanda & 7 & 0,8 \\
Cuba & 6 & 0,7 \\
Argentina & 5 & 0,5 \\
\hline
\end{tabular}




\section{Periódicos com maior número de publicações}

Neste item foram identificados os periódicos utilizados para publicação de pesquisas de autores brasileiros na área de construção e tecnologia da construção. Esse indicador é importante por mostrar os principais periódicos científicos da área, fonte de disseminação do conhecimento mais utilizada pelos pesquisadores, e importante canal de comunicação científica (SILVA, 2002).

O periódico com maior número de artigos publicados com autoria de pesquisadores brasileiros é o Construction and Building Materials(n=116 artigos, $12,7 \%$ do total da produção brasileira sobre Construction \& Building Technology na WoS). Dos 15 periódicos com maior número de artigos publicados, seis tratam do tema Materiais(Construction and Building Materials, Materials and Structures,Journal of Materials in Civil Engineering, ACI Materials Journal,Materiales de Construcción,Road Materials and Pavement Design), três tratam mais especificamente do tema Concreto (Cement Concrete Composites, Cement and Concrete Research, Magazine of Concrete Research), dois tratam do tema Estruturas (Materials and Structures, Journal of Constructional Steel Research, Journal of Structural Engineering, ACI
Structural Journal), e os demais tratam de Energia e Meio Ambiente. Esses cinco temas parecem ser os principais temas de pesquisas desenvolvidas por brasileiros, com resultados divulgados em periódicos internacionais (Tabela 4).

Entre as categorias definidas pela WoS, $100 \%$ dos artigos estão classificados na categoria Construction Building Technology, critério utilizado para seleção, mas também estão classificados em outras categorias, sendo engenharia civil a categoria mais ampla, que engloba a construção civil, a segunda com maior número de artigos associados $(\mathrm{n}=513,56,4 \%)$, seguida de materiais (ciência dos materiais e composição), energia e meio ambiente, o que confirma os temas associados aos títulos dos principais periódicos.

A Tabela 5 apresenta os principais eventos científicos indexados na base WoS, em que os pesquisadores brasileiros divulgam suas pesquisas na área de construção e tecnologia da construção. $\mathrm{O}$ evento com maior destaque foi específico sobre energia solar $(n=13$ artigos, $1,4 \%$ da produção brasileira na área). Entre os cinco principais eventos, nota-se a preferência por eventos temáticos, tais como materiais (pedras e madeiras), estruturas e energia.

Tabela 4 - Frequência da produção científica brasileira em construção e tecnologia da construção por periódico indexado na WoS no período de 1982 a 2014

\begin{tabular}{l|c|c}
\hline \multicolumn{1}{c|}{ Periódicos } & Artigos & \% \\
\hline Construction and Building Materials & 116 & 12,7 \\
Cement Concrete Composites & 70 & 7,7 \\
Cement and Concrete Research & 61 & 6,7 \\
Energy and Buildings & 60 & 6,6 \\
Materials and Structures & 56 & 6,2 \\
Journal of Constructional Steel Research & 54 & 5,9 \\
Building and Environment & 49 & 5,4 \\
Journal of Materials in Civil Engineering & 22 & 2,4 \\
Journal of Structural Engineering ASCE & 22 & 2,4 \\
ACI Materials Journal & 16 & 1,8 \\
Materiales de Construcción & 13 & 1,4 \\
Road Materials and Pavement Design & 13 & 1,4 \\
HVAC R Research & 11 & 1,2 \\
ACI Structural Journal & 10 & 1,1 \\
Magazine of Concrete Research & 10 & 1,1 \\
\hline
\end{tabular}

Fonte: Web of Science (2014). 
Tabela 5 - Eventos científicos indexados na WoS com maior número de produções científicas brasileiras em construção e tecnologia da construção no período de 1982 a 2014

\begin{tabular}{l|r|r}
\hline \multicolumn{1}{c|}{ Eventos Científicos } & Artigos & \% \\
\hline Solar World Congress of the International Solar Energy Society & 13 & 1,4 \\
Conference of the International Building Performance Simulation Association & 11 & 1,2 \\
International RILEM Symposium on Timber Engineering & 8 & 0,9 \\
International Conference on Structures and Architecture ICSA & 7 & 0,8 \\
Global Stone Congress & 7 & 0,8 \\
\hline
\end{tabular}

Fonte: Web of Science (2014).

\section{Discussão dos resultados da pesquisa}

A partir da análise da produção científica brasileira de construção e tecnologia da construção realizada neste estudo, com base em dados indexados na base de dados da Web of Science, suportada pela utilização de técnicas bibliométricas, foi possível identificar um crescimento significativo, apresentando um avanço constante do número de publicações. Esse fato indica que a área está se fortalecendo e aumentando sua atuação em pesquisa científica. No entanto, o crescimento ocorrido pode ser considerado como discreto quando comparado com a relevância do setor de construção e tecnologia da construção para o desenvolvimento social, econômico e seu papel no crescimento do produto interno bruto (PIB) do país.

Em relação à localização geográfica da produção científica no Brasil, a região Sudeste foi a que mais contribui com publicações na área de construção e tecnologia da construção. Entre as instituições científicas, a Universidade de São Paulo (USP), Universidade Federal do Rio de Janeiro (UFRJ), Universidade Federal de Santa Catarina (UFSC), Universidade Federal do Rio Grande do Sul (UFRGS), Universidade Federal de Minas Gerais (UFMG), Pontifícia Universidade Católica do Rio de Janeiro (PUC-RJ) e Universidade Estadual de Campinas (Unicamp) foram as instituições de ensino que mais publicaram.

Esses resultados provavelmente estão relacionados à contínua evolução dos programas de pósgraduação para a formação de recursos humanos, além de receber mais recursos financeiros dos órgãos oficiais de fomento à Pesquisa \& Desenvolvimento, visando à geração e à difusão do conhecimento na área de construção e tecnologia da construção.

Quanto às áreas temáticas mais publicadas pelos autores brasileiros destacam-se a área de engenharia, com $61 \%$, e a área de materiais, com $55 \%$ dos artigos associados. As demais áreas energia e combustíveis, ciência da computação, arquitetura, geologia, termodinâmica, saúde ocupacional, entre outras - ratificam a diversidade da cadeia produtiva da construção civil e sua interação com outras áreas do conhecimento. Vale ressaltar que a produção científica brasileira por autor com maior número de publicações indexadas na WoS está vinculada tanto às instituições públicas (UFRJ, USP e UERJ) como às privadas (PUC-PR e PUC-RJ)

Os periódicos que apresentaram maior número de publicação pelos pesquisadores brasileiros em construção civil foram o Construction and Building Materials, Cement and Concrete Composites, Cement and Concrete Research e Energy and Building.

Nesse contexto, as técnicas bibliométricas mostram que a pesquisa pode ser de grande utilidade para a descoberta da produção científica na área de construção e tecnologia da construção. Os dados contidos neste estudo podem ser utilizados como ferramenta tanto para o planejamento como para a execução de políticas, visando melhor compreender a ciência pela comunidade científica, empresarial e outros segmentos da sociedade.

\section{Referências}

BANCO NACIONAL DE
DESENVOLVIMENTO ECONÔMICO E SOCIAL. Fundo de Estruturação de Projetos: BNDES FEP. 2010. Disponível em: <http://www.bndes.gov.br/SiteBNDES/bndes/bnde s_pt/Institucional/Apoio_Financeiro/Apoio_a_estu dos_e_pesquisas/BNDES_FEP/index.html>. Acesso em: 03 nov. 2015.

CÂMARA BRASILEIRA DA INDÚSTRIA DA CONSTRUÇÃO. Indicadores da Conjuntura Nacional e Setorial.Resumo Contas Nacionais: PIB Brasil e Construção Civil. Disponível em: $<$ http://www.cbicdados.com.br/menu/pib-einvestimento/pib-brasil-e-construcao-civil>. Acesso em: 25 set. 2013. 
CENTRO DE GESTÃO E ESTUDOS ESTRATÉGICOS. Relatório Prospectivo Setorial: construção civil. Brasília, 2009.

DEPARTAMENTO DA INDÚSTRIA DA CONSTRUÇÃO FIESP. Proposta de Política Industrial Para Construção Civil: edificações. São Paulo: FIESP, out. 2008.

FUNDAÇÃO DE AMPARO À PESQUISA DO ESTADO DE SÃO PAULO. Indicadores de Ciência, Tecnologia e Inovação em São Paulo 2004. São Paulo: Fapesp, 2005.

FUNDAÇÃO DE AMPARO À PESQUISA DO ESTADO DE SÃO PAULO. Indicadores de Ciência, Tecnologia e Inovação em São Paulo 2010. São Paulo: Fapesp, 2010.

GREGOLIN, J. A. R. et al. Análise da Produção Científica a Partir de Indicadores Nibliométricos. In: FUNDAÇÃO DE AMPARO À PESQUISA DO ESTADO DE SÃO PAULO. Indicadores de Ciência, Tecnologia e Inovação em São Paulo 2004. São Paulo: Fapesp, 2005. cap. 5, p. 1-44.

MARTINS, M. G.; BARROS, M. M. S. B.

AFormação de Parcerias Como Alternativa Para Impulsionar a Inovação na Produção de Edifícios. In: SIMPÓSIO BRASILEIRO DE GESTÃO E ECONOMIA NA CONSTRUÇÃO, 3., São Carlos, 2003. Anais...São Carlos, 2003.

PROGRAMA INOVAÇÃO TECNOLÓGICA NA CONSTRUÇÃO. [Construção]. Disponível em: <http://www.pit.org.br/1a-fase/construcao>. Acesso em: 10 jun. 2012.

RAO, I. K. Métodos Quantitativos em Biblioteconomia e em Ciência da Informação. Brasília: ABDF, 1986.
REHEN, S. Blog Pode Ser Futuro da Publicação Científica. G1, 18 abr. 2007. Disponível em: <http://g1.globo.com/Noticias/Ciencia/0,,MUL235 99-5603,00.html>. Acesso em: 28 jul. 2012.

SILVA, S. Comunicação Organizacional em Empresas de Construção Civil Sob a Ótica do Planejamento Estratégico. 157 f. Curitiba, 2002. Dissertação (Mestrado em Construção Civil) Programa de Pós-Graduação em Construção Civil, Universidade Federal do Paraná, Curitiba, 2002.

SOUTO, M. S. M. L. Estudo Comparativo Entre Brasil e Portugal das Práticas Gerenciais em Empresas de Construção Civil Sob a Ótica da Gestão do Conhecimento. Florianópolis, 2003. Tese (Doutorado em Engenharia de Produção) Programa de Pós-Graduação em Engenharia de Produção, Universidade Federal de Santa Catarina, Florianópolis, 2003.

SU, H.; LEE, P. Mapping Knowledge Structure by Keyword Co-Occurrence: a first look at journal papers in technology foresight. Scientometrics, v. 85, n. 1, p.65-79, jun. 2010.

THOMSON REUTERS. Brasil: atuais desafios e tendências da inovação. Set. 2013. Disponívelem: $<$ http://www.inpi.gov.br/images/docs/brazil__current_innovation_trends_ and_challenges_final_091313_pt-br.pdf>. Acesso em: 22 dez. 2013.

\section{VIOTTI, E. B. Indicadores de Inovação}

Tecnológica: fundamentos, evolução e sua situação no Brasil. Brasília: Ministério do Desenvolvimento, Indústria e Comércio Exterior, 2001.

ZHU, D.et al.A Process For Mining Science \& Technology DocumentsDatabaseIllustrate For the Case ofKnowledge Discovery and Data Mining. Ciência da Informação, v. 28, n. 1, jan. 1999. 


\section{Patrícia Bourguignon Soares}

Departamento de Engenharia Civil, Centro Tecnológico | Universidade Federal do Espírito Santo | Av. Fernando Ferrari, s/n, Campus Universitário, Goiabeiras | Vitória - ES - Brasil | CEP 29060-900 | Tel.: (27) 3335-2174 | E-mail: patricia.copes@yahoo.com.br

\section{Teresa Cristina Janes Carneiro}

Departamento de Administração, Centro de Ciências Jurídicas e Econômicas | Universidade Federal do Espírito Santo | Tel.: (27) 4009-2096 | E-mail: carneiro.teresa@gmail.com

\section{João Luiz Calmon}

Departamento de Engenharia Civil, Centro Tecnológico | Universidade Federal do Espírito Santo \Tel.: (27) 3335-2174 |

E-mail: calmonbarcelona@gmail.com

\section{Luiz Otávio da Cruz de Oliveira Castro}

Departamento de Engenharia Civil, Centro Tecnológico | Universidade Federal do Espírito Santo | Tel.: (27)3335-2174 |

E-mail: lotavioc@gmail.com

\section{Revista Ambiente Construído}

Associação Nacional de Tecnologia do Ambiente Construído

Av. Osvaldo Aranha, $99-3^{\circ}$ andar, Centro

Porto Alegre - RS - Brasil

CEP 90035-190

Telefone: +55 (51) 3308-4084

Fax: +55 (51) 3308-4054

www.seer.ufrgs.br/ambienteconstruido

E-mail: ambienteconstruido@ufrgs.br 